\title{
Survey on Auction based Scheduling in Grid and Cloud Environment
}

\author{
Santhiya $\mathrm{H}$ \\ Post Graduate Scholar \\ Department of Computer Science and Engineering \\ Karunya University, India
}

\author{
Karthikeyan P \\ Assistant Professor \\ Department of Computer Science and Engineering \\ Karunya University, India
}

\begin{abstract}
Grid and Cloud Computing allow the user to execute applications on a third-party infrastructure by paying for the access to the remote resources. Scheduling and resource management is an important aspect for effective execution of an application. This paper addresses various auction based scheduling in grid and cloud environments. To optimize the user centric and provider centric objectives the market based auction models are used. An auction is an economic mechanism for allocating the resources among a group of users. The benefits achieved by the resource users and resource providers are different for various auction models. This paper shows that Continuous Double Auction model is efficient and favour for both resource users and resource providers.
\end{abstract}

\section{General Terms}

Auction, Grid.

\section{Keywords}

Resource Management, Double Auction, Scheduling, Market Model, Grid/Cloud computing.

\section{INTRODUCTION}

Computational grid it supports for the creation of virtual organizations it allows for sharing, selection and aggregation of distributed heterogeneous resources. The resource users can use the resources by running the grid portals and also the resource providers can share the resources by using the grid portals. The grid users can access the resources based on their requirements and demands and also based on the availability of the resources. Normally the grid environment is dynamic in nature at any moment the resource providers with various resources and services can add or removed from the grid, and also the resource user with various demands can enter into the grid.

The resources and end users are geographically distributed among different time zones [1]. The resources are dynamic and heterogeneous in nature, sharing and managing the resources in such an environment is a challenging task .In Grid environments both resource users and resource providers having different goals, objectives, strategies and policies.The user centric objectives are execution time and execution cost. The provider centric objectives are allocation of resources, income and job throughput. Scheduler is responsible for allocating the resources among a group of resource users. When the multiple users competing for the same resource then scarcity of particular resource will occur and also it assumes the other users as competitors. Since the access for the resource is paid, it can be seen as a market governed by the supply-demand law. Economy-based negotiation techniques are attractive from the point of view of business .Both resource users and providers are tries to get the profit of their own. In an economy-based approach [2], during the run time the scheduling decisions will be taken based on the end user requirements. The economy model charges the end user based on their usage of services. The pricing policies which is based on the demand of end-user and the supply of the resources. It creates the competitive environment. This is the reason the users competes with other users and the resource providers competes with other providers. The end users are not willing to pay the highest price so they negotiate on the price based on their need, priority and budget.

Nowadays market based methods get more attention for grid resource management. The main two classification of grid resource management is commodity market model and auction model [8]. The commodity market model, in which the resource owner will fix the rate for the resources and charge the users according to the amount of resources they use. It is based on flat price model, once the price is fixed then it does not changed its price based on supply and demand, it remain fixed for a certain period of time. The resource owner is responsible for fixing and changing the price of the resources. The auction model is different from commodity market model, in which both the user and the resource provider will act separately and they agree on the selling price. In auction model it has no fixed value or price and it affected by a supply and demand law at any specific period of time. If the need of resource is low then they will decrease the price of the resource. If the demand is high then they will increase the price of the particular resource. The interaction between the user and resource provider takes place as a negotiation process it expressed in terms of service level agreement

The paper has been classified as follows. Section 2 discuss about concept of basic auction models. Section 3 discuss about the classification on of auction models. Section 4 discuss about continuous double auction model.

\section{AUCTION MODEL}

Auction model [3] is the powerful model for allocating the resources in the grid environment. Normally auction means it is a mechanism for allocating the resources among a group of bidders. Auctions can be conducted as open or closed form. The buyer will update the bid and the seller will update the offered price. Each auction has three different entities the seller, the buyer and the auctioneer. The auctioneer is responsible for the overall management of the auction. The interaction between the entities during an auction is shown in Figure 1. 


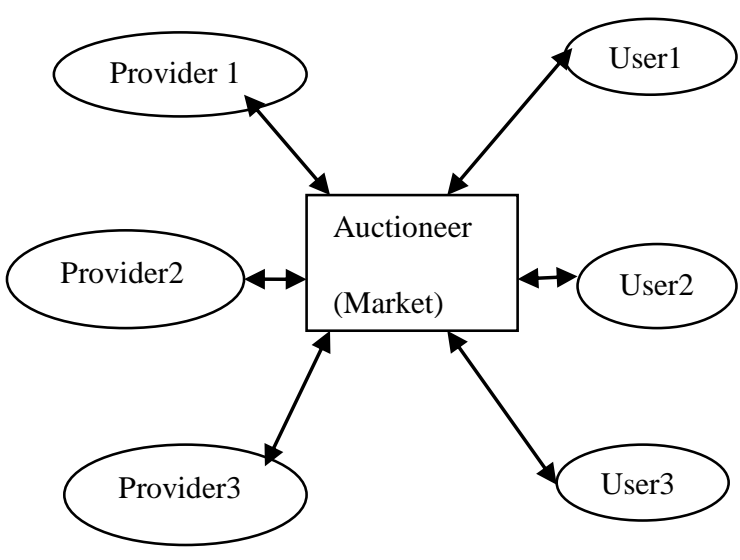

Fig 1: Interaction between entities during action

\section{CLASSIFICATION OF AUCTION MODELS}

The auction models are classifieds into two main categories, single sided and double sided auction. Fig2 shows the different classification of auction models.

In single sided auctions [5], once the resources are submitted by the sellers then the buyers only submit their offers during the auction. The single sided auctions are English Auction, Dutch Auction, First price sealed Auction, Vickrey Auction.

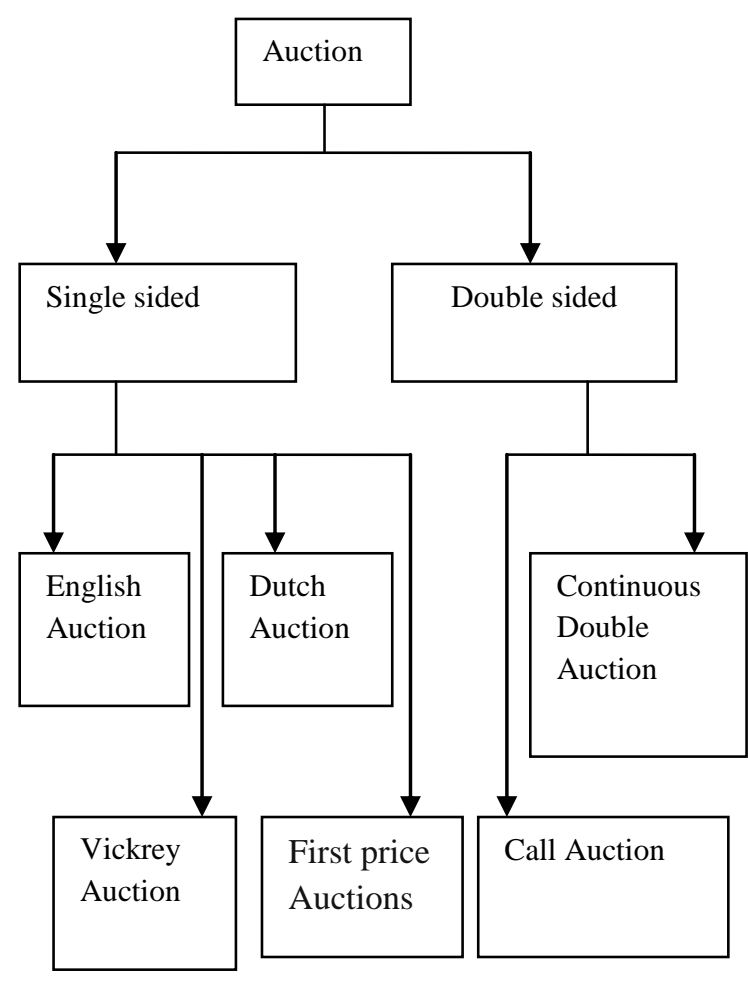

Fig 2: Classification of Auction Models

\subsection{English Auction}

In English Auction the seller will fix the price for the resource then buyers will constantly increase their bids, until the buyer with highest bid wins the auction. The English Auction is an open auction model so the bidders will sensibly bid for the resources. The drawback of this auction model is it requires higher demand of communication. The price charged for the resources is too high. Understanding the market condition and judging the value for the resources is difficult. The time required for computational time is higher in the English Auction.

\subsection{Dutch Auction}

In Dutch auction the seller will fix the highest price for the resource and continuously decrease the price until the buyer is ready to pay for the resource. It is similar to English auction. When the demand for the service is high then the bidders will bid earlier so the communication demand is low. If the demand for the service is low then the bidders will not bid until the price drops so the communication demands is high. The demand of communication is high or less based on the demand of the resources. It required less computational time than the English Auction. It also requires the knowledge of the market and identifying the correct value for the resource is difficult.

\subsection{First Price Sealed Auction}

In First Price Sealed Auction the buyers submit their bids in the envelope without knowing the others bid, the buyer with the highest bid will wins in the auction. The buyers pay the exact amount which they mentioned in the envelope. It will decrease the sudden raise of the price. It is better for the resource providers.

\subsection{Vickery Auction}

In Vickery Auction which is similar to the First Price Sealed Auction the buyers have no idea about the bid value of other bidders. The highest bidders win the auction and pay the amount which is equal to the second highest bid. The Vickery Auction is better from the perspective of user.

\subsection{Double Auction}

In Double Auction both the buyer and the seller will submit their offers which are known as bid and ask. The Double Auction [10] is classifieds into two main types, Call Auction and Continuous Double Auction.

In Continuous Double Auction (CDA) both the buyer and seller will submit their bid and ask continuously until the match between the ask and bid occur. The time spent for the computation is less. It require lower level of communication

In Call Auction the auction has a predefined time frame when the participants submit their offer.

\section{CONTINUOUS DOUBLE AUCTION MODEL}

In continuous double auction model [9] both the buyer and sellers will submit their offers continuously until the match between the lowest ask and highest bid occur. When a match between the lowest asks $a_{\text {min }}$ and the highest bid $b_{\max }$ is achieved, the user who submits the highest bid will win the auction. The final price the user who wins the auction has to pay is $\frac{\left(a_{\min }+b_{\max }\right)}{2}$, it is the final outcome of the auction. The user who wins the auction will use the service for the specified time. The others participating in auction lose it. If the auction won by any user then the auction is also won by a particular resource provider. If the auction is cancelled then the resource provider loses the auction. When multiple users competing for the same resource then insufficiency of resources will occur. Both the user and the resource providers always try optimizing their own objectives so conflicts will 
occur. So negotiation process will take place in between the resource user and the resource providers.

The main actors of resource allocation process are users and the resource providers. The user submit their bids and the resource provider submit their asks to the central market (Auctioneer).In the central market the actual auction will take place. It is responsible for sending the results of the auction to the actors. Fig3 shows the entire structure of Continuous Double Auction (CDA).

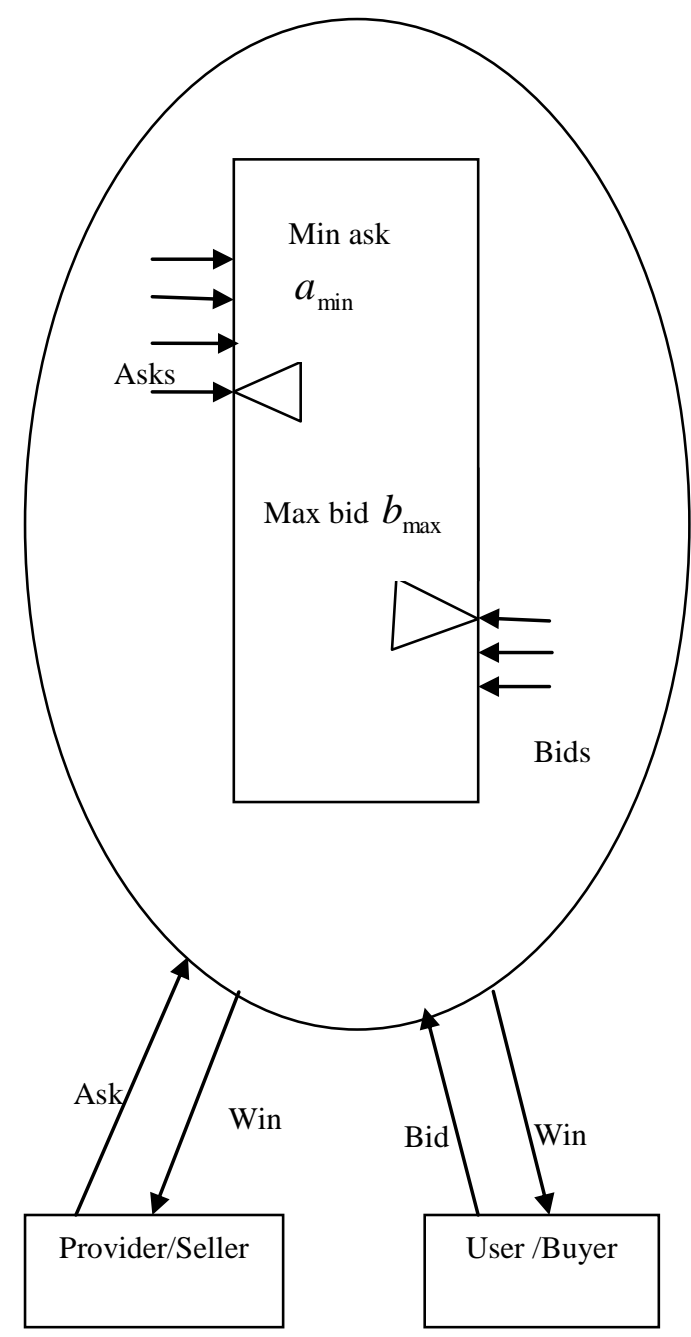

Fig 3: Structure of CDA

The step by step which is performed in the continuous double auction model is as follows

- The resource provider creates the auction and initiates the auction wit initial price

- User will continuously submit their bids and tries to win the resource during the auction.

- Then the resource provider submits their asks and increase the price of the resources.

- When the match between the highest ask and lowest bid occur, the user who submit the highest bid will win the auction.
- If no match is detected then the auction closed without the winner.

- The auctioneer sends the winning notifications to the winner and the resource provider.

- The auctioneer sends the loss notification to the other users who participated in the auction.

- The user who wins the auction will execute the service.

In continuous double auction model, once the match is detected then immediately allocates the resources to the user. The matching bid and the ask is deleted from the auction. And also the result is informed to the user and the provider by the auctioneer. If the auction failed then it will start the auction after a certain period of delay time interval.

The resources are priced based on their supply and demand. When the demand for the resource is high then they will increase the price, if the demand is less then they will decrease the price. The main objective of the user is to spend less money and the provider is to gain more profit. It can check based on three different networks [7][6].

In Balanced network the total number of tasks and the resources are equal so there is no price growth or drops occur. It's in normal price no changes happen because the demand and supply is equal.

In task intensive network there is large number of tasks than the resources. It shows that the demand for the resource is high and the supply is low. When this condition occurs then automatically sudden price growth will occur.

In resource intensive network there is large number of tasks than the resources. It shows that the demand for the resources is low and the supply is high. Then the price will be decreased. Based on these conditions the price for the resources will be grown up or fall down.

When comparing the continuous double auction model with the other auction models. In English Auction the multiple bidders will bid within a single round. So the number of messages exchange will be higher. It is favour for users. In Dutch auction the bidders do not bid until they are interested in price so the number of messages exchanged will be low compared to English Auction. Only the number of rounds will be varying but the final price is similar. In first price sealed auction number of rounds will be lower, it provide lower benefit for users. It is favour for resource providers. But the vickrey auction which is favour for users. In CDA [4] it requires only the lesser amount of communication and it is favour for both resource users and the providers. Table 1 shows the comparison between auction models. Each model is different and it shows the computation time, communication demand and profit of different auction models. 
Table 1. Comparison of various Auction Models

\begin{tabular}{|c|c|c|c|}
\hline Auction models & $\begin{array}{c}\text { Computation } \\
\text { time }\end{array}$ & $\begin{array}{c}\text { Communication } \\
\text { demand }\end{array}$ & Profit \\
\hline English Auction & High & Higher & Provider \\
\hline Dutch Auction & Medium & Medium & Provider \\
\hline $\begin{array}{c}\text { First Sealed Auction } \\
\text { Lickrey Auction }\end{array}$ & Low & Less & Provider \\
\hline Continuous Double & & Less & User \\
\hline Auction & Very Low & Very low & Both user and Provider \\
\hline
\end{tabular}

\section{CONCLUSION}

Thus the analysis of auction based scheduling and the classification of auction models is discussed. The Continuous Double Auction model which is favour for both resource users and the providers. It requires less computation time and the demand for communication is low. It is an efficient model for resource management in grid and cloud environment.

\section{REFERENCES}

[1] Abramson, D., Buyya, R., Giddy, J. (2 002) "A computational economy for Grid computing and its implementation in the nimrod-g resource broker". Future Gener. Comput. Syst. ,1061-1074

[2] Buyya, R., Abramson, D., Venugopal, S. (2005) "The Grid economy" Proc. I.E.E.E. 698-714 .

[3] Buyya, R., Stockinger, H., Giddy, J., Abramson, D. (2001) "Economic models for resource management and scheduling in Grid computing",Journal of Grid Computing, 171-200 .

[4] Dias de Assunção, M., Buyya, R. (2006) "An evaluation of communication demand of auction protocols in Grid environments". 3rd International Workshop on Grid Economics and Business Models. World Scientific Publications.
[5] Hesam Izakian, Behrouz Tork Ladani, Kamran Zamanifar, Ajith Abraham and Vaclav Snasel. (2002) "A Continuous Double Auction Method for Resource Allocation in Computational Grids" The Journal of Concurrency and Computation, 1507 - 1542.

[6] P. K. Suri , and Manpreet Singh .) (2009) "Auction Oriented Resource Allocation in Grid Using Knowledge Based Pricing Policy". International Journal of Computer Science and Network Security

[7] Pourebrahimi, , S., Alima, L. (2005) “A dynamic pricing and bidding strategy for autonomous agents in Grids". International Workshop on Agents and Peer-to-Peer

[8] Pourebrahimi, B., Bertels, K., Kandru, G.M., Vassiliadis, S. (2006) "Market-based resource allocation in Grids". 2nd International Conference on e-Science and Grid Computing.

[9] Prodan, R., Wieczorek, M., Mohammadi, H. (2011) "Double Auction-based Scheduling of ScientificApplications in Distributed Grid and Cloud Environments". Journal of Grid Computing 9:531-548.

[10] Tan, Z., Gurd, J.R.: Market-based Grid resource allocation using a stable continuous double auction. In: $8^{\text {th }}$ International Conference on Grid Computing. IEEE Computer Society Press, Austin, Texas, USA (2007). 